\title{
Anomalous and topological Hall effects in epitaxial thin films of the noncollinear antiferromagnet $\mathrm{Mn}_{3} \mathrm{Sn}$
}

\author{
James M. Taylor $\odot,{ }^{1, *}$ Anastasios Markou $\odot,{ }^{2}$ Edouard Lesne, ${ }^{1}$ Pranava Keerthi Sivakumar $\odot,{ }^{1}$ Chen Luo $\odot,{ }^{3}$ \\ Florin Radu $\odot,{ }^{3}$ Peter Werner, ${ }^{1}$ Claudia Felser $\odot,{ }^{2}$ and Stuart S. P. Parkin $\odot^{1, \dagger}$ \\ ${ }^{1}$ Max Planck Institute of Microstructure Physics, Weinberg 2, 06120 Halle (Saale), Germany \\ ${ }^{2}$ Max Planck Institute for Chemical Physics of Solids, Nöthnitzer Str. 40, 01187 Dresden, Germany \\ ${ }^{3}$ Helmholtz-Zentrum Berlin for Materials and Energy, Albert Einstein Str. 15, 12489 Berlin, Germany
}

(Received 9 October 2019; accepted 13 January 2020; published 2 March 2020)

\begin{abstract}
Noncollinear antiferromagnets with a $D 0_{19}$ (space group $\left.=194, P 6_{3} / m m c\right)$ hexagonal structure have garnered much attention for their potential applications in topological spintronics. Here, we report the deposition of continuous epitaxial thin films of such a material, $\mathrm{Mn}_{3} \mathrm{Sn}$, and characterize their crystal structure using a combination of x-ray diffraction and transmission electron microscopy. Growth of $\mathrm{Mn}_{3} \mathrm{Sn}$ films with both (0001) $c$-axis orientation and $(40 \overline{4} 3)$ texture is achieved. In the latter case, the thin films exhibit a small uncompensated Mn moment in the basal plane, quantified via magnetometry and $\mathrm{x}$-ray magnetic circular dichroism experiments. This cannot account for the large anomalous Hall effect simultaneously observed in these films, even at room temperature, with magnitude $\sigma_{x y}\left(\mu_{0} H=0 \mathrm{~T}\right)=21 \Omega^{-1} \mathrm{~cm}^{-1}$ and coercive field $\mu_{0} H_{c}=1.3 \mathrm{~T}$. We attribute the origin of this anomalous Hall effect to momentum-space Berry curvature arising from the symmetry-breaking inverse triangular spin structure of $\mathrm{Mn}_{3} \mathrm{Sn}$. Upon cooling through the transition to a glassy ferromagnetic state at around $50 \mathrm{~K}$, a peak in the Hall resistivity close to the coercive field emerges. This indicates the onset of a topological Hall effect contribution, arising from a nonzero scalar spin chirality that generates a real-space Berry phase. We demonstrate that the polarity of this topological Hall effect, and hence the chiral nature of the noncoplanar magnetic structure driving it, can be controlled using different field-cooling conditions.
\end{abstract}

DOI: 10.1103/PhysRevB.101.094404

\section{INTRODUCTION}

Antiferromagnets (AF) are of interest for spintronic applications [1]; however, they struggle to produce readout signals of the size required for devices. Large magnetotransport signatures, such as the intrinsic anomalous Hall effect (AHE) [2], generated by topological materials may offer the solution [3]. In particular, topological noncollinear AFs of the form $\mathrm{Mn}_{3} X$ are of interest.

$\mathrm{Mn}_{3} \mathrm{Sn}$ is a noncollinear AF with Mn moments arranged in hexagonal crystal planes [4], which exhibits an inverse triangular spin structure resulting from a combination of exchange and Dzyaloshinskii-Moriya (DM) interactions [5]. The inverse triangular AF order breaks time-reversal symmetry, thus introducing momentum-space Berry curvature. In turn, this induces a fictitious magnetic field, which has been theoretically predicted [6] to drive a highly anisotropic AHE [7]. This was subsequently experimentally measured in single crystals of hexagonal $\mathrm{Mn}_{3} \mathrm{Sn}$ [8] and $\mathrm{Mn}_{3} \mathrm{Ge}$ [9].

\footnotetext{
*james.taylor@mpi-halle.mpg.de

${ }^{\dagger}$ stuart.parkin@mpi-halle.mpg.de
}

Published by the American Physical Society under the terms of the Creative Commons Attribution 4.0 International license. Further distribution of this work must maintain attribution to the author(s) and the published article's title, journal citation, and DOI. Open access publication funded by the Max Planck Society.
The emergence of Berry curvature is connected with Weyl points arising in $\mathrm{Mn}_{3} \mathrm{Sn}$ [10-12]. The presence of such Weyl quasiparticles close to the Fermi level also induces an anomalous Nernst effect [13-15]. Further enhancement of the attractiveness of $\mathrm{Mn}_{3} \mathrm{Sn}$ for spintronics stems from its hosting of a Berry curvature-driven intrinsic spin Hall effect [16-18], which was originally discovered in the cubic noncollinear $\mathrm{AF}$ $\mathrm{Mn}_{3} \operatorname{Ir}[19]$.

The symmetry-breaking inverse triangular spin texture also gives rise to a magneto-optical Kerr effect [20-22]. Kerr microscopy imaging of this effect reveals that $\mathrm{Mn}_{3} \mathrm{Sn}$ contains $\mathrm{AF}$ domains possessing opposite chiralities of the noncollinear spin structure. These reversed chiralities correspond to opposite signs of a cluster octupole order parameter [23] and, hence, reversed polarities of magnetotransport properties across different AF domains [24].

An external magnetic field can be used to propagate $\mathrm{AF}$ domains of a particular chirality [22], in order to generate a net magnetotransport output. The applied magnetic field couples to the small uncompensated magnetic moment [25] that is created in the hexagonal crystal planes of $\mathrm{Mn}_{3} \mathrm{Sn}$ by spins spontaneously canting slightly towards magnetocrystalline easy axes [26]. This weak magnetization can freely rotate within the basal plane [5], in turn acting to orient the entire inverse triangular spin texture, and, when saturated, achieving a single chiral domain state.

As $\mathrm{Mn}_{3} \mathrm{Sn}$ is cooled, its magnetic order changes depending on microstructure [27]. At $275 \mathrm{~K}$, Mn-deficient samples 


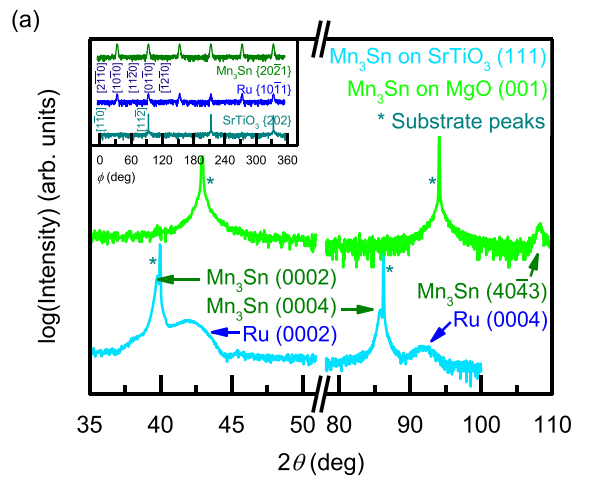

(d)

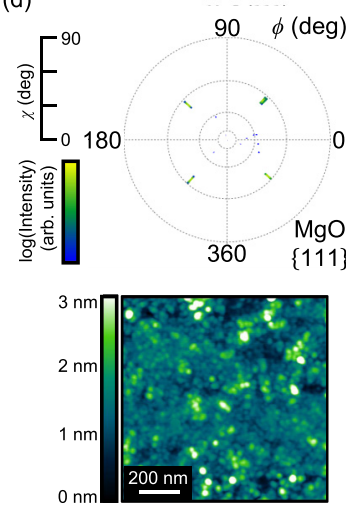

(b)

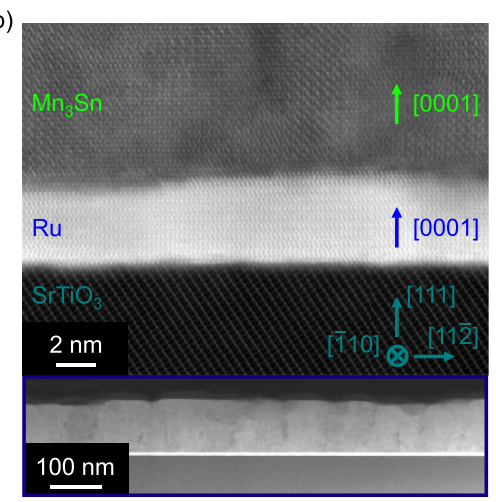

(e)

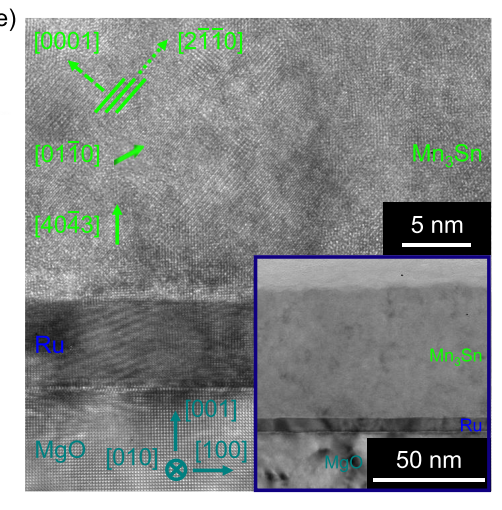

(c)

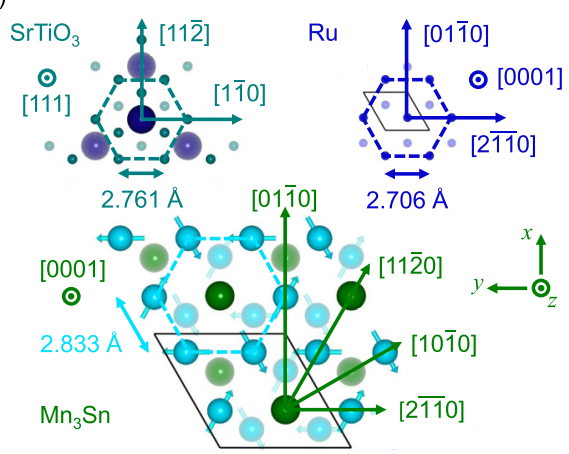

(f)
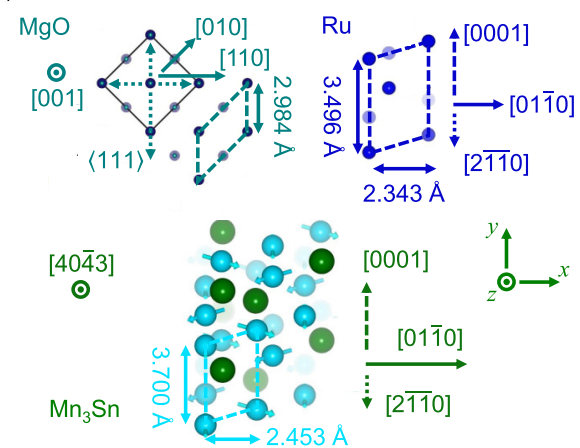

FIG. 1. (a) $2 \theta-\theta$ x-ray diffraction scans measured for a 70-nm $\mathrm{Mn}_{3} \mathrm{Sn}(0001)$ film grown on a $\mathrm{SrTiO}_{3}$ (111) substrate, and for a 60-nm $\mathrm{Mn}_{3} \mathrm{Sn}(40 \overline{4} 3)$ film grown on a $\mathrm{MgO}(001)$ substrate. Inset shows azimuthal $\phi$ scans of the $\mathrm{SrTiO}_{3}\{202\}, \operatorname{Ru}\{10 \overline{1} 1\}$, and $\mathrm{Mn}{ }_{3} \mathrm{Sn}\{20 \overline{2} 1\}$ reflections for a 70-nm Mn $\mathrm{Mn}_{3} \mathrm{Sn}$ (0001) sample. The corresponding positions of in-plane crystallographic directions are indicated. (b) Crosssection scanning-TEM micrograph of a 70-nm $\mathrm{Mn}_{3} \mathrm{Sn}$ (0001) film, viewed along the [110] zone axis of the $\mathrm{SrTiO}_{3}(111)$ substrate. Inset shows a wide-view image over an extended region of the same lamella. (c) Representative crystal structure of $\mathrm{Mn}_{3} \mathrm{Sn}(0001)$ films grown on $\mathrm{SrTiO}_{3}$ (111) substrates using a Ru buffer layer. (d) $\chi-\phi$ x-ray pole figures measured for a $60-\mathrm{nm} \mathrm{Mn}_{3} \mathrm{Sn}(40 \overline{4} 3$ ) sample. The MgO [100] crystalline axis was aligned with $\phi=0^{\circ}$. Inset shows an atomic force microscopy topographic map for a similar 50-nm $\mathrm{Mn}_{3} \mathrm{Sn}(40 \overline{4} 3)$ film, capped with

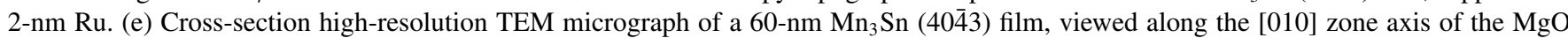
(001) substrate. Inset shows an overview TEM image measured for the same sample. (f) Representative crystal structure of $\mathrm{Mn}_{3} \mathrm{Sn}_{\mathrm{n}}(40 \overline{4} 3)$ films grown on $\mathrm{MgO}$ (001) substrates using a Ru buffer layer.

transition to a helical magnetic phase [28]. Around $50 \mathrm{~K}$, the magnetic structure changes to a "glassy" ferromagnetic (FM) (or spin-glass) state [29], regardless of composition, in which Mn moments are frustrated to cant out of the basal planes [30]. Studies in bulk samples [31] show this is accompanied by the onset of a topological Hall effect (THE) [32], attributed to a possible magnetic skyrmion phase [33], or to chiral domain walls [34].

Recently, AHE has been measured in polycrystalline $\mathrm{Mn}_{3} \mathrm{Sn}$ films [35-37]. In addition, a planar Hall effect has been observed in epitaxial films [38]. In this paper, we extend these previous results by studying the structural and magnetic properties of epitaxial $\mathrm{Mn}_{3} \mathrm{Sn}$ thin films, and demonstrating the presence of both AHE and THE.

\section{RESULTS AND DISCUSSION}

\section{A. Film growth and structural characterization}

We previously grew $\mathrm{Mn}_{3} \mathrm{Sn}$ films that were epitaxial but discontinuous [39]. By changing substrate and optimizing postannealing temperature, we succeeded in fabricating continuous $\mathrm{Mn}_{3} \mathrm{Sn}$ films (details in the Supplemental Material [40]). Figure 1(a) shows $2 \theta-\theta$ x-ray diffraction (XRD) patterns measured for films deposited on $\mathrm{SrTiO}_{3}$ (111) and $\mathrm{MgO}$ (001) substrates. In both cases, a 5-nm Ru buffer layer was used [39]. The presence of $\{0002\}$ diffraction peaks indicates the growth of $c$-axis oriented films on $\mathrm{SrTiO}_{3}$ (111) substrates. Lattice parameters calculated from these XRD measurements establish that these hexagonal $\mathrm{Mn}_{3} \mathrm{Sn}$ (0001) films grow fully relaxed (analysis in the Supplemental Material [40]). Azimuthal XRD $\phi$ scans of partially in-plane (IP) peaks, shown in the inset of Fig. 1(a), demonstrate the same epitaxial relationship as in Ref. [39]: $\mathrm{SrTiO}_{3}(111)[11 \overline{2}]$ [11̄0]\| $\operatorname{Ru}(0001)[01 \overline{1} 0][2 \overline{110}] \| \mathrm{Mn}_{3} \operatorname{Sn}(0001)[01 \overline{1} 0][2110]$, depicted schematically in Fig. 1(c).

We studied film structure at the nanoscale using transmission electron microscopy (TEM). Figure 1(b) shows a cross-section scanning-TEM micrograph from a 70-nm film deposited on $\mathrm{SrTiO}_{3}$ (111), which demonstrates explicitly the epitaxial growth of (0001)-oriented $\mathrm{Mn}_{3} \mathrm{Sn}$ on a $\mathrm{Ru}(0001)$ buffer. The inset of Fig. 1(b) displays a wide-view scanningTEM image of the same lamella, confirming the preparation of continuous films. 
Figure 1(a) also shows a single peak in the $2 \theta-\theta$ XRD scan for a 60-nm film deposited on $\mathrm{MgO}(001)$; this corresponds

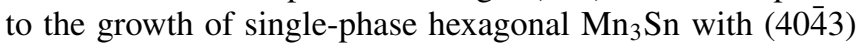
orientation. We analyzed this crystal texture by recording $\chi-\phi$ XRD pole-figure maps around the expected positions of the \{111\} reflections of $\mathrm{MgO},\{0002\}$ reflections of $\mathrm{Ru}$, and $\{0002\}$ reflections of $\mathrm{Mn}_{3} \mathrm{Sn}$, as presented in Fig. 1(d). The $\{0002\}$ reflections of $\mathrm{Ru}$ follow the cubic $\{111\} \mathrm{MgO}$ peaks, indicating that the hexagonal planes of $\mathrm{Ru}$ are tilted to lie at the same angle as the (111) planes of the substrate. The $\{0002\}$ reflections of $\mathrm{Mn}_{3} \mathrm{Sn}$ in turn follow the Ru diffraction peaks, confirming that the basal planes of $\mathrm{Mn}_{3} \mathrm{Sn}$ are seeded to grow at the same angle as those in the buffer layer. This results in a structure for $\mathrm{Mn}_{3} \mathrm{Sn}(40 \overline{4} 3)$ with the [0001] crystalline direction at almost $55^{\circ}$ to the film normal, the [2110] magnetic easy axis [26] at approximately $35^{\circ}$ to the film normal, and the [0110] crystallographic axis lying completely IP. This epitaxial relationship is illustrated in Fig. 1(f).

The $\mathrm{Mn}_{3} \mathrm{Sn}\{0002\}$ reflections are fourfold symmetric. This indicates four distinct crystallite orientations in which the $c$ axis, and in turn the orthogonal [0110] direction, follows one of the possible IP $\langle 110\rangle \mathrm{MgO}$ axes. Therefore, for a chosen macroscopic measurement direction, there will be equivalent numbers of $\mathrm{Mn}_{3} \mathrm{Sn}$ crystal grains with either the [0001] or [0110] axes parallel to this.

Nevertheless, high-resolution TEM of an individual $\mathrm{Mn}_{3} \mathrm{Sn}(40 \overline{4} 3$ ) crystallite, as depicted in Fig. 1(e), confirms single-crystalline growth. The [01 10$]$ crystallographic axis is directed at $45^{\circ}$ into the plane of the image. The basal planes of $\mathrm{Mn}_{3} \mathrm{Sn}$ are clearly visible, while the [2 $\overline{11} 0$ ] magnetic easy axis is aligned almost out of plane (OP). That these crystal grains coherently coalesce to form a continuous thin film is ascertained from wide-view TEM images, an example of which is shown in the inset of Fig. 1(e).

Finally, we used atomic force microscopy to quantify the roughness of $\mathrm{Mn}_{3} \mathrm{Sn}$. An example topographic map is shown in the inset of Fig. 1(d), which yields an average roughness of

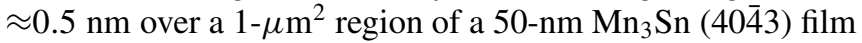
capped with 2-nm Ru.

\section{B. Magnetic properties}

Figure 2(a) shows the magnetization $(M)$ of a $60-\mathrm{nm}$ $\mathrm{Mn}_{3} \mathrm{Sn}(40 \overline{4} 3)$ film measured using superconducting quantum interference device vibrating sample magnetometry (SQUIDVSM, Quantum Design MPMS3), after subtracting the background contribution from a $\mathrm{MgO}$ substrate/5-nm Ru reference sample. When magnetic field $\left(\mu_{0} H\right)$ is applied OP, with a component along the [2 $\overline{110}$ ] easy axis, an opening of the loop in the region $\pm 1.5 \mathrm{~T}$ is attributed to the reversal of the small uncompensated moment expected in the basal plane of $\mathrm{Mn}_{3} \mathrm{Sn}$.

With magnetic field applied along one of two orthogonal IP directions, a similar hysteretic behavior is observed, but with a smaller magnitude and an isotropic response. This is because magnetization is averaged over many crystallites, which can be fourfold symmetrically oriented with either [0110] (in the plane where uncompensated moment freely rotates) or partially IP [0001] (hard axis) directions parallel to the magnetic field.

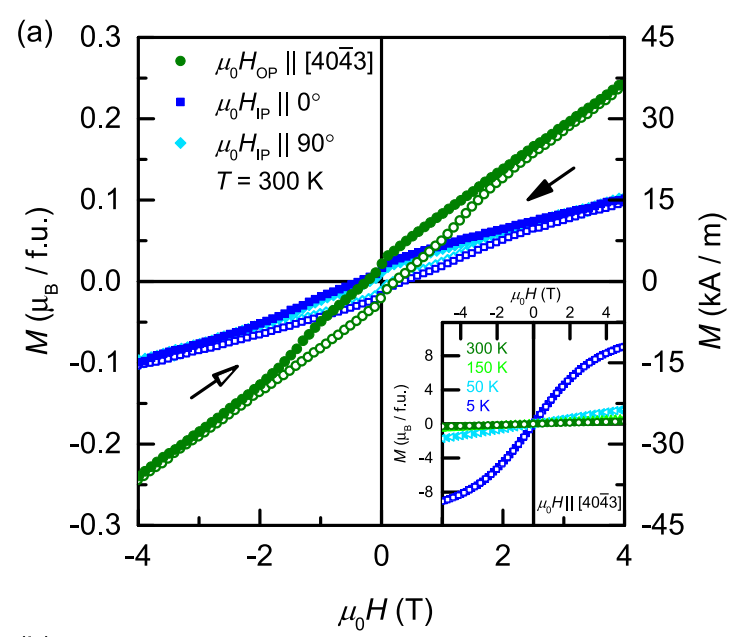

(b)

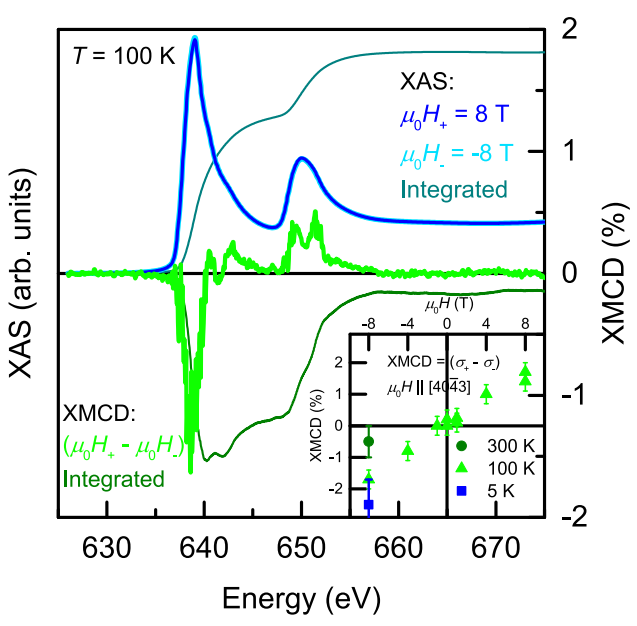

FIG. 2. (a) Magnetization measured as a function of magnetic field for a $60-\mathrm{nm} \mathrm{Mn}_{3} \mathrm{Sn}(40 \overline{4} 3)$ film at $300 \mathrm{~K}$. Magnetic field was applied either out of plane or along one of two orthogonal in-plane directions (closed and open symbols represent down and up field sweeps, respectively). Inset shows magnetization as a function of out-of-plane magnetic field, measured at different temperatures. (b) X-ray absorption spectroscopy and XMCD spectra for a 70-nm

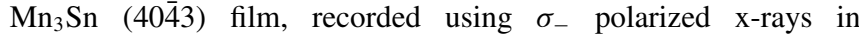
$\mu_{0} H_{ \pm}= \pm 8 \mathrm{~T}$ magnetic fields applied out of plane, at $100 \mathrm{~K}$. Inset shows XMCD, calculated as the difference between spectra recorded with $\sigma_{ \pm}$polarized X-rays, measured in different out-ofplane magnetic fields at $100 \mathrm{~K}$, as well as that measured in $-8 \mathrm{~T}$ at 300 and $5 \mathrm{~K}$.

The inset of Fig. 2(a) plots magnetization response to magnetic field at different temperatures $(T)$. At $5 \mathrm{~K}$, a significant enhancement of magnetization demonstrates the appearance of the glassy FM phase [29].

$\mathrm{X}$-ray magnetic circular dichroism (XMCD) was measured at the BESSY synchrotron facility [41], using negative, $\sigma_{-}$, polarized $\mathrm{x}$ rays in an OP magnetic field of $\mu_{0} H_{ \pm}= \pm 8 \mathrm{~T}$. Nonzero XMCD around the $\mathrm{Mn} L_{2,3}$ edges at $100 \mathrm{~K}$, displayed in Fig. 2(b), confirms the presence of a net Mn moment that is reversible by external magnetic field. Using XMCD sum-rule analysis [42], an uncompensated magnetic moment of $m_{\mathrm{s}+1}=0.279 \mu_{\mathrm{B}} /$ f.u. was calculated, comprising spin, $m_{\mathrm{s}}=0.273 \mu_{\mathrm{B}} /$ f.u., and orbital, $m_{\mathrm{l}}=0.006 \mu_{\mathrm{B}} /$ f.u. moments respectively. Measured $m_{1}$ is of the same order of 
magnitude as that simulated [25], while $m_{\mathrm{s}}$ is found to be substantially larger than the theoretically predicted value for zero-field weak magnetization.

We explain this by considering the inset of Fig. 2(b), showing the magnetic-field dependence of XMCD, measured as in Ref. [43]. XMCD increases approximately linearly, because of Mn spins tilting out of the film plane in response to strong applied magnetic fields [43]. This therefore enhances $m_{\mathrm{s}}$, whose magnitude is in agreement with that determined from SQUID-VSM, which indicates similar paramagnetic behavior after the closing of the hysteresis loop.

Nevertheless, both measurements reveal that the uncompensated moment overall is enhanced compared with bulk crystals, including the remnant weak magnetization within the basal plane measured at zero-applied magnetic field using SQUID-VSM. This uncompensated moment is exacerbated because of structural defects and chemical disorder present in the thin films, which may act to modify the balance of AF exchange, DM interactions, and magnetocrystalline anisotropy, thus increasing the spontaneous canting of Mn spins [39].

The inset of Fig. 2(b) also presents XMCD measured in a -8 -T magnetic field at different temperatures. XMCD is reduced at $300 \mathrm{~K}$, while its enhancement at $5 \mathrm{~K}$ evidences an increased net moment after transition to the spin-glass state.

\section{Magnetotransport measurements}

We now report magnetotransport measurements in $\mathrm{Mn}_{3} \mathrm{Sn}$ thin films lithographically patterned into $75 \times 25-\mu \mathrm{m}^{2}$ Hall bar devices. Figure 3(a) shows the variation in longitudinal resistivity $\left(\rho_{\mathrm{xx}}\right)$ for a 70 -nm-thick $\mathrm{Mn}_{3} \mathrm{Sn}(0001)$ film as a function of temperature, during either zero-field cooling (ZFC) or cooling in a 7-T OP magnetic field (FC), followed in both cases by zero-field warming (ZFW). A background contribution from the 5-nm Ru buffer layer has been subtracted (see Supplemental Fig. S2 [40]). On cooling, a deviation from metallic behavior below $100 \mathrm{~K}$, down to a bump in resistivity close to $50 \mathrm{~K}$, provides evidence for the transition to the glassy FM phase. No change is observed between ZFC and FC protocols, as expected with cooling field parallel to the [0001] hard axis.

During subsequent ZFW, a thermal hysteresis is seen. Resistivity first drops between 50 and $100 \mathrm{~K}$. The resulting difference in resistivity is maintained, as above $100 \mathrm{~K}$ resistivity rises at the same rate compared to ZFC but with a smaller absolute value, until the film is warmed above room temperature. This may indicate that the transition from the inverse triangular to glassy FM state that occurs on cooling is not fully reversible until the thin film reaches close to the Néel temperature of $\mathrm{Mn}_{3} \mathrm{Sn}\left(T_{\mathrm{N}}=420 \mathrm{~K}\right.$ [4]). This can be explained if regions of the spin-glass structure become pinned at defects via their chiral domain walls (discussed below).

Figure 3(b) plots the variation in transverse resistivity $\left(\rho_{x y}\right)$ as a function of OP magnetic field for the same 70-nm $\mathrm{Mn}_{3} \mathrm{Sn}$ (0001) film. At room temperature, we observe a linear Hall effect. This is because external field is applied along the $c$ axis, which is a magnetic hard axis. Therefore, applied field is unable to manipulate the weak magnetization, which occurs within the basal plane, and is thus unable to drive the (a)
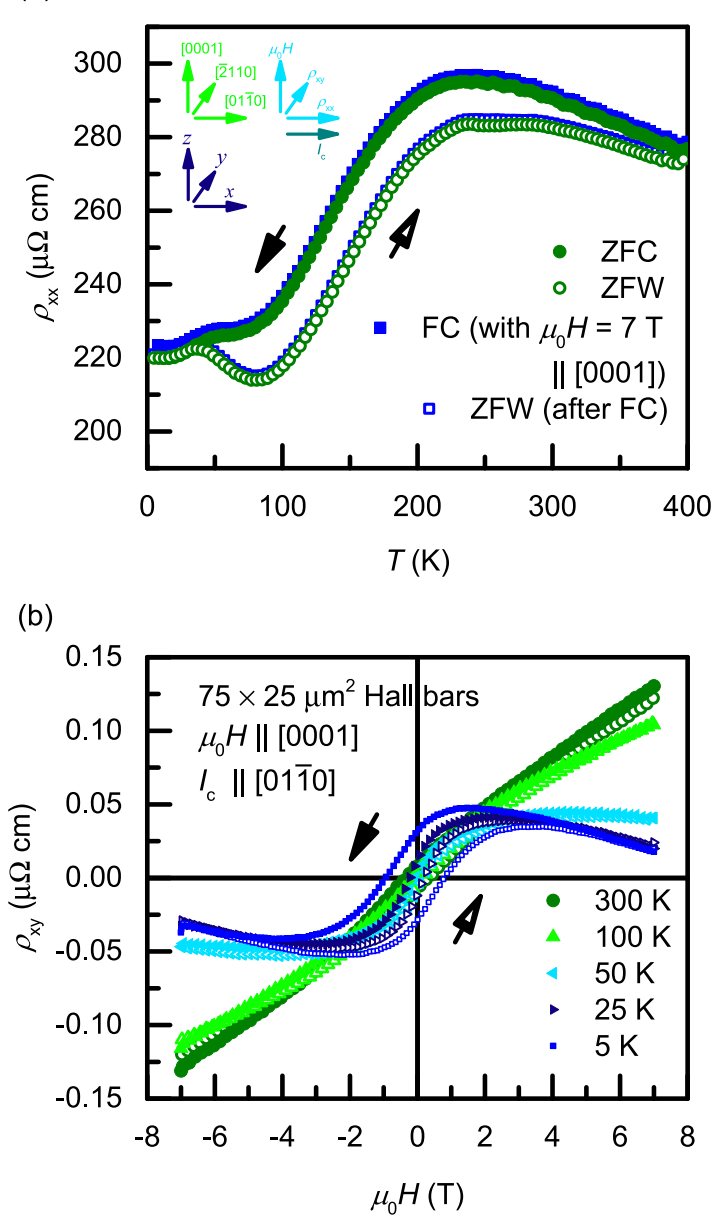

FIG. 3. Magnetotransport for a 70-nm $\mathrm{Mn}_{3} \mathrm{Sn}$ (0001) film patterned into $75 \times 25-\mu \mathrm{m}^{2}$ Hall bars, with $500-\mu \mathrm{A}$ current parallel to the $[01 \overline{1} 0]$ in-plane crystallographic direction. (a) Longitudinal resistivity measured as a function of temperature during either zerofield cooling, or cooling in a 7-T magnetic field applied out of plane (closed symbols), and subsequent zero-field warming (open symbols). Diagram illustrates measurement geometry in relation to different crystallographic directions in the $\mathrm{Mn}_{3} \mathrm{Sn}$ (0001) film. (b) Hall resistivity measured as a function of magnetic field applied out of plane at different temperatures. Transverse resistivity was measured along the [ $\overline{2} 110]$ crystalline axis (closed and open symbols represent down and up field sweeps, respectively).

thin film into a dominant chiral domain state (which would yield a net contribution to Berry curvature-induced AHE). Between 100 and $50 \mathrm{~K}$, this ordinary Hall effect changes to an AHE as $\mathrm{Mn}_{3} \mathrm{Sn}$ transitions into the glassy FM phase. Here, moments are frustrated to cant along the [0001] axis [30], with the resulting FM-like component of magnetization producing a conventional AHE in response to an OP magnetic field.

Moving onto the $\mathrm{Mn}_{3} \mathrm{Sn}(40 \overline{4} 3)$ films, we sourced current $\left(I_{c}\right)$ along the $[01 \overline{1} 0]$ direction and measured a component of Hall resistivity partially orthogonal to the (0001) crystal planes. Magnetic field was applied parallel to the [40"43]

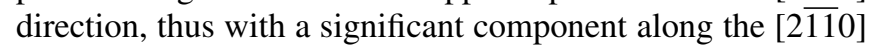
easy axis. A schematic of this measurement configuration is inset in Fig. 4(b). These crystalline directions are as defined in 
(a)

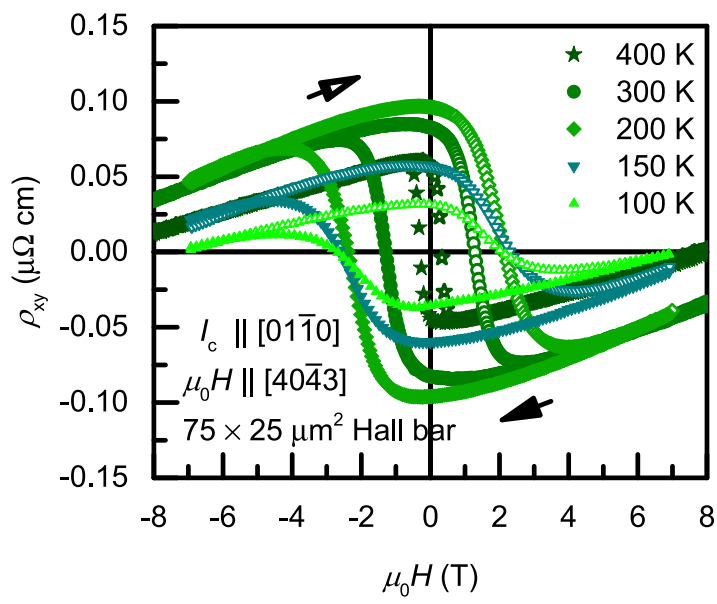

(b)

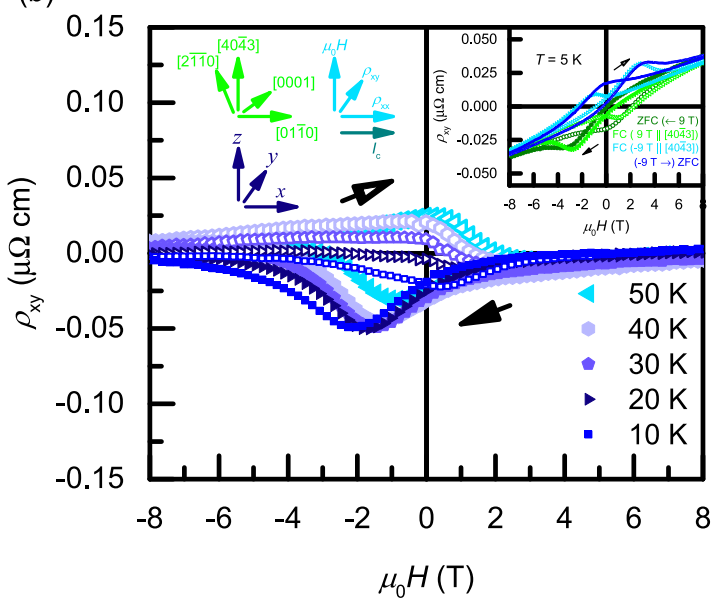

FIG. 4. Magnetotransport for a $60-\mathrm{nm} \mathrm{Mn}_{3} \mathrm{Sn}(40 \overline{4} 3)$ film patterned into $75 \times 25-\mu \mathrm{m}^{2}$ Hall bars. Transverse resistivity was measured normal to the (0001) crystal plane, with $500-\mu \mathrm{A}$ current parallel to the [01 10$]$ in-plane crystallographic direction (closed and open symbols represent down and up field sweeps, respectively). Isothermal Hall resistivity measured as a function of magnetic field applied out of plane in the (a) higher-temperature regime and (b) lower-temperature regime. Diagram illustrates measurement geometry in relation to different crystallographic directions in the

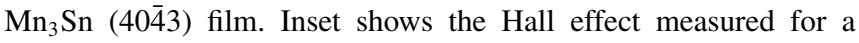
30-nm $\mathrm{Mn}_{3} \mathrm{Sn}(40 \overline{4} 3)$ film at $5 \mathrm{~K}$, after cooling under different field conditions.

one of the four equivalent structural grains (see Supplemental Fig. S5 [40]).

Figures 4(a) and 4(b) show isothermal measurements of

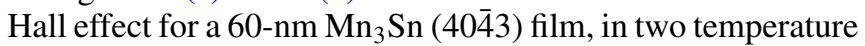
regimes. At room temperature, we measure a large AHE driven by momentum-space Berry curvature [8]. A tiny longitudinal magnetoresistance $(<0.02 \%$ at $8 \mathrm{~T})$ is simultaneously observed (see Supplemental Material [40]), ruling out FM contributions to magnetotransport.

Consistent AHE is measured across a series of $\mathrm{Mn}_{3} \mathrm{Sn}$

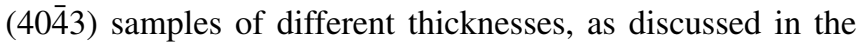
Supplemental Material [40]. We extract a remnant anomalous Hall conductivity, $\sigma_{x y}\left(\mu_{0} H=0 \mathrm{~T}\right)=21 \Omega^{-1} \mathrm{~cm}^{-1}$, comparable to previous reports [35].
Figure 4(a) shows that as temperature is increased towards $T_{\mathrm{N}}$, coercive field decreases because of a softening of the magnetic structure. However, as temperature is decreased below $100 \mathrm{~K}$, the magnitude of the Berry curvature-generated AHE drops concomitantly, as $\mathrm{Mn}_{3} \mathrm{Sn}$ begins to leave the inverse triangular AF phase.

Below $50 \mathrm{~K}, \mathrm{Mn}_{3} \mathrm{Sn}$ transitions into the glassy FM state, where momentum-space Berry curvature-driven and (in this measurement configuration) conventional AHE are no longer detected. Instead, as shown in Fig. 4(b), the hysteresis loop becomes prominently asymmetric; a signature of the THE, caused when electrons acquire a real-space Berry phase upon encountering a magnetic structure with nonzero scalar spin chirality [32].

The inset of Fig. 4(b) explores this THE in a $30-\mathrm{nm} \mathrm{Mn}_{3} \mathrm{Sn}$

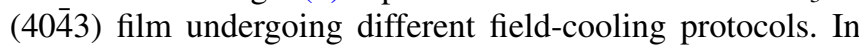
the first case, the sample is saturated in a 9-T OP magnetic field, setting a particular chirality of the inverse triangular spin texture, which is maintained as the field is decreased to zero. The film is then cooled (ZFC). Here a downward bump arising close to the point of magnetization reversal (when external field is swept from positive to negative) indicates a negative THE. A bump with the same negative polarity is seen at coercivity on the reverse (negative to positive) field sweep. This demonstrates the formation of a noncollinear, noncoplanar spin texture close to the coercive field, at which point magnetic order is reversing through a mechanism of domain nucleation and propagation.

Therefore, we attribute the THE to the nucleation of chiral domain walls. Mn moments forming the domain walls will rotate within the basal plane, in order to invert magnetic structure between one domain and the next [31]. A domain wall with such arrangement will appear as a noncollinear, noncoplanar spin texture possessing finite scalar spin chirality to electrons propagating within the plane of these $\mathrm{Mn}_{3} \mathrm{Sn}$

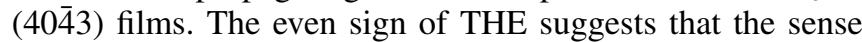
of rotation of spins in the domain walls is opposite during forward and reverse magnetization processes, while the internal moment configuration within the domain walls remains the same.

These results are in agreement with Li et al. [34], who measure a symmetric planar Hall effect in $\mathrm{Mn}_{3} \mathrm{Sn}$ single crystals. This is attributed to chiral domain walls with a fixed internal moment configuration, which is maintained during both positive to negative, and negative to positive, field sweeps. The sense of rotation of spins within the domain walls must therefore be inverted between forward and reverse field sweeps. They also observe a dependence of the planar Hall effect polarity on applied field history.

We measure a similar magnetic state history in $\mathrm{Mn}_{3} \mathrm{Sn}$ (40) 3 ) films. The inset of Fig. 4(b) shows that in the second case, when the thin film is ZFC after saturation in a -9-T OP magnetic field, the polarities of both symmetric THE peaks invert. This represents a sign change of realspace Berry phase, corresponding to an opposite sign of the finite scalar spin chirality generating it. We propose, therefore, that the handedness of the inverse triangular spin texture set at room temperature favors a certain chirality of domain wall formed during low-temperature magnetization reversal. 
Furthermore, the THE bumps with either negative or positive polarity are enhanced after cooling in a $+9-\mathrm{T}$ or $-9-\mathrm{T}$ OP magnetic field, respectively. This may be explained by the external field further stabilizing the preferred domainwall moment configuration during cooling. We thus demonstrate a memory effect in noncollinear AF thin films, that can be controlled by setting the orientation of the inverse triangular spin texture from which the chirality of domain walls evolves, which may find applications in neuromorphic computing.

\section{CONCLUSION}

In conclusion, we have grown epitaxial thin films of $\mathrm{Mn}_{3} \mathrm{Sn}$

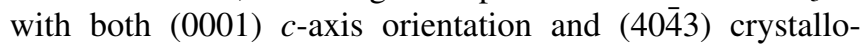
graphic structure. In the latter case, Berry curvature-driven AHE is observed at room temperature. Upon cooling through the magnetic phase transition at $50 \mathrm{~K}$, a peak in the Hall resistivity indicates the appearance of a THE. The sign of this THE signal, and hence the chirality of the noncoplanar spin texture generating it, can be manipulated through fieldcooling conditions, thus furthering the potential of $\mathrm{Mn}_{3} \mathrm{Sn}$ in chiralitronic devices.

\section{ACKNOWLEDGMENTS}

We acknowledge Dr. Dominik Kriegner for preparation

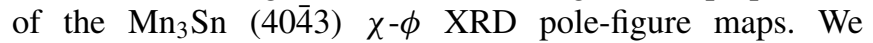
thank Prof. Christian Back for provision of synchrotron beamtime at Helmholtz-Zentrum Berlin on beamline PM2 VEKMAG (under Proposal No. 191-07803), and acknowledge Wolfgang Simeth and Denis Mettus for assistance with measurements. Financial support for the VEKMAG project and for the PM2 VEKMAG beamline is provided by the German Federal Ministry for Education and Research (BMBF Contracts No. 05K10PC2, No. 05K10WR1, and No. 05K10KE1) and by Helmholtz-Zentrum Berlin, with Steffen Rudorff thanked for technical support. This work was partially funded by ASPIN (EU H2020 FET Open Grant No. 766566).
[1] V. Baltz, A. Manchon, M. Tsoi, T. Moriyama, T. Ono, and Y. Tserkovnyak, Antiferromagnetic spintronics, Rev. Mod. Phys. 90, 015005 (2018).

[2] N. Nagaosa, J. Sinova, S. Onoda, A. H. MacDonald, and N. P. Ong, Anomalous Hall effect, Rev. Mod. Phys. 82, 1539 (2010).

[3] L. Šmejkal, Y. Mokrousov, B. Yan, and A. H. MacDonald, Topological antiferromagnetic spintronics, Nat. Phys. 14, 242 (2018).

[4] P. J. Brown, V. Nunez, F. Tasset, J. B. Forsyth, and P. Radhakrishna, Determination of the magnetic structure of $\mathrm{Mn}_{3} \mathrm{Sn}$ using generalized neutron polarization analysis, J. Phys.: Condens. Matter 2, 9409 (1990).

[5] S. Tomiyoshi and Y. Yamaguchi, Magnetic structure and weak ferromagnetism of $\mathrm{Mn}_{3} \mathrm{Sn}$ studied by polarized neutron diffraction, J. Phys. Soc. Jpn. 51, 2478 (1982).

[6] J. Kübler and C. Felser, Non-collinear antiferromagnets and the anomalous Hall effect, Europhys. Lett. 108, 67001 (2014).

[7] Y. Zhang, Y. Sun, H. Yang, J. Železný, S. S. P. Parkin, C. Felser, and B. Yan, Strong anisotropic anomalous Hall effect and spin Hall effect in the chiral antiferromagnetic compounds $\mathrm{Mn}_{3} \mathrm{X}$ (X = Ge, Sn, Ga, Ir, Rh, and Pt), Phys. Rev. B 95, 075128 (2017).

[8] S. Nakatsuji, N. Kiyohara, and T. Higo, Large anomalous Hall effect in a non-collinear antiferromagnet at room temperature, Nature (London) 527, 212 (2015).

[9] A. K. Nayak, J. E. Fischer, Y. Sun, B. Yan, J. Karel, A. C. Komarek, C. Shekhar, N. Kumar, W. Schnelle, J. Kübler, C. Felser, and S. S. P. Parkin, Large anomalous Hall effect driven by a nonvanishing Berry curvature in the noncolinear antiferromagnet $\mathrm{Mn}_{3} \mathrm{Ge}$, Sci. Adv. 2, e1501870 (2016).

[10] H. Yang, Y. Sun, Y. Zhang, W.-J. Shi, S. S. P. Parkin, and B. Yan, Topological Weyl semimetals in the chiral antiferromagnetic materials $\mathrm{Mn}_{3} \mathrm{Ge}$ and $\mathrm{Mn}_{3} \mathrm{Sn}$, New J. Phys. 19, 015008 (2017).

[11] J. Kübler and C. Felser, Weyl fermions in antiferromagnetic $\mathrm{Mn}_{3} \mathrm{Sn}$ and $\mathrm{Mn}_{3} \mathrm{Ge}$, Europhys. Lett. 120, 47002 (2017).
[12] K. Kuroda, T. Tomita, M. T. Suzuki, C. Bareille, A. A. Nugroho, P. Goswami, M. Ochi, M. Ikhlas, M. Nakayama, S. Akebi, R. Noguchi, R. Ishii, N. Inami, K. Ono, H. Kumigashira, A. Varykhalov, T. Muro, T. Koretsune, R. Arita, S. Shin, T. Kondo, and S. Nakatsuji, Evidence for magnetic Weyl fermions in a correlated metal, Nat. Mater. 16, 1090 (2017).

[13] M. Ikhlas, T. Tomita, T. Koretsune, M.-T. Suzuki, D. NishioHamane, R. Arita, Y. Otani, and S. Nakatsuji, Large anomalous Nernst effect at room temperature in a chiral antiferromagnet, Nat. Phys. 13, 1085 (2017).

[14] X. Li, L. Xu, L. Ding, J. Wang, M. Shen, X. Lu, Z. Zhu, and K. Behnia, Anomalous Nernst and Righi-Leduc Effects in $\mathrm{Mn}_{3} \mathrm{Sn}$ : Berry Curvature and Entropy Flow, Phys. Rev. Lett. 119, 056601 (2017).

[15] C. Wuttke, F. Caglieris, S. Sykora, F. Scaravaggi, A. U. B. Wolter, K. Manna, V. Süss, C. Shekhar, C. Felser, B. Büchner, and C. Hess, Berry curvature unravelled by the anomalous Nernst effect in $\mathrm{Mn}_{3} \mathrm{Ge}$, Phys. Rev. B 100, 085111 (2019).

[16] Y. Zhang, J. Železný, Y. Sun, J. v. d. Brink, and B. Yan, Spin Hall effect emerging from a noncollinear magnetic lattice without spin-orbit coupling, New J. Phys. 20, 073028 (2018).

[17] M. Kimata, H. Chen, K. Kondou, S. Sugimoto, P. K. Muduli, M. Ikhlas, Y. Omori, T. Tomita, A. H. MacDonald, S. Nakatsuji, and Y. Otani, Magnetic and magnetic inverse spin Hall effects in a non-collinear antiferromagnet, Nature (London) 565, 627 (2019).

[18] P. K. Muduli, T. Higo, T. Nishikawa, D. Qu, H. Isshiki, K. Kondou, D. Nishio-Hamane, S. Nakatsuji, and Y. Otani, Evaluation of spin diffusion length and spin Hall angle of the antiferromagnetic Weyl semimetal $\mathrm{Mn}_{3} \mathrm{Sn}$, Phys. Rev. B 99, 184425 (2019).

[19] W. Zhang, W. Han, S. H. Yang, Y. Sun, Y. Zhang, B. Yan, and S. S. P. Parkin, Giant facet-dependent spin-orbit torque and spin Hall conductivity in the triangular antiferromagnet $\mathrm{IrMn}_{3}$, Sci. Adv. 2, e1600759 (2016). 
[20] W. Feng, G.-Y. Guo, J. Zhou, Y. Yao, and Q. Niu, Large magneto-optical Kerr effect in noncollinear antiferromagnets $\mathrm{Mn}_{3} \mathrm{X}(\mathrm{X}=\mathrm{Rh}, \mathrm{Ir}, \mathrm{Pt})$, Phys. Rev. B 92, 144426 (2015).

[21] A. L. Balk, N. H. Sung, S. M. Thomas, P. F. S. Rosa, R. D. McDonald, J. D. Thompson, E. D. Bauer, F. Ronning, and S. A. Crooker, Comparing the anomalous Hall effect and the magneto-optical Kerr effect through antiferromagnetic phase transitions in $\mathrm{Mn}_{3} \mathrm{Sn}$, Appl. Phys. Lett. 114, 032401 (2019).

[22] T. Higo, H. Man, D. B. Gopman, L. Wu, T. Koretsune, O. M. J. van't Erve, Y. P. Kabanov, D. Rees, Y. Li, M. T. Suzuki, S. Patankar, M. Ikhlas, C. L. Chien, R. Arita, R. D. Shull, J. Orenstein, and S. Nakatsuji, Large magneto-optical Kerr effect and imaging of magnetic octupole domains in an antiferromagnetic metal, Nat. Photon. 12, 73 (2018).

[23] M. T. Suzuki, T. Koretsune, M. Ochi, and R. Arita, Cluster multipole theory for anomalous Hall effect in antiferromagnets, Phys. Rev. B 95, 094406 (2017).

[24] H. Reichlová, T. Janda, J. Godinho, A. Markou, D. Kriegner, R. Schlitz, J. Železný, Z. Soban, M. Bejarano, H. Schultheiss, P. Němec, T. Jungwirth, C. Felser, J. Wunderlich, and S. T. B. Goennenwein, Imaging and writing magnetic domains in the non-collinear antiferromagnet $\mathrm{Mn}_{3} \mathrm{Sn}$, Nat. Commun. 10, 5459 (2019).

[25] L. M. Sandratskii and J. Kübler, Role of Orbital Polarization in Weak Ferromagnetism, Phys. Rev. Lett. 76, 4963 (1996).

[26] T. F. Duan, W. J. Ren, W. L. Liu, S. J. Li, W. Liu, and Z. D. Zhang, Magnetic anisotropy of single-crystalline $\mathrm{Mn}_{3} \mathrm{Sn}$ in triangular and helix-phase states, Appl. Phys. Lett. 107, 082403 (2015).

[27] E. Krén, J. Paitz, G. Zimmer, and É. Zsoldos, Study of the magnetic phase transformation in the $\mathrm{Mn}_{3} \mathrm{Sn}$ phase, Physica B 80, 226 (1975).

[28] N. H. Sung, F. Ronning, J. D. Thompson, and E. D. Bauer, Magnetic phase dependence of the anomalous Hall effect in $\mathrm{Mn}_{3}$ Sn single crystals, Appl. Phys. Lett. 112, 132406 (2018).

[29] W. J. Feng, D. Li, W. J. Ren, Y. B. Li, W. F. Li, J. Li, Y. Q. Zhang, and Z. D. Zhang, Glassy ferromagnetism in $\mathrm{Ni}_{3}$ Sn-type $\mathrm{Mn}_{3.1} \mathrm{Sn}_{0.9}$, Phys. Rev. B 73, 205105 (2006).

[30] S. Tomiyoshi, S. Abe, Y. Yamaguchi, H. Yamauchi, and H. Yamamoto, Triangular spin structure and weak ferromagnetism of $\mathrm{Mn}_{3} \mathrm{Sn}$ at low temperature, J. Magn. Magn. Mater. 54-57, 1001 (1986).

[31] X. Li, L. Xu, H. Zuo, A. Subedi, Z. Zhu, and K. Behnia, Momentum-space and real-space Berry curvatures in $\mathrm{Mn}_{3} \mathrm{Sn}$, SciPost Phys. 5, 063 (2018).
[32] C. Sürgers, G. Fischer, P. Winkel, and H. v. Löhneysen, Large topological Hall effect in the non-collinear phase of an antiferromagnet, Nat. Commun. 5, 3400 (2014).

[33] P. K. Rout, P. V. P. Madduri, S. K. Manna, and A. K. Nayak, Field-induced topological Hall effect in the noncoplanar triangular antiferromagnetic geometry of $\mathrm{Mn}_{3} \mathrm{Sn}$, Phys. Rev. B 99, 094430 (2019).

[34] X. Li, C. Collignon, L. Xu, H. Zuo, A. Cavanna, U. Gennser, D. Mailly, B. Fauqué, L. Balents, Z. Zhu, and K. Behnia, Chiral domain walls of $\mathrm{Mn}_{3} \mathrm{Sn}$ and their memory, Nat. Commun. 10, 3021 (2019).

[35] T. Ikeda, M. Tsunoda, M. Oogane, S. Oh, T. Morita, and Y. Ando, Anomalous Hall effect in polycrystalline $\mathrm{Mn}_{3} \mathrm{Sn}$ thin films, Appl. Phys. Lett. 113, 222405 (2018).

[36] T. Higo, D. Qu, Y. Li, C. L. Chien, Y. Otani, and S. Nakatsuji, Anomalous Hall effect in thin films of the Weyl antiferromagnet $\mathrm{Mn}_{3} \mathrm{Sn}$, Appl. Phys. Lett. 113, 202402 (2018).

[37] T. Ikeda, M. Tsunoda, M. Oogane, S. Oh, T. Morita, and Y. Ando, Improvement of large anomalous Hall effect in polycrystalline antiferromagnetic $\mathrm{Mn}_{3+x} \mathrm{Sn}$ thin films, IEEE Trans. Magn. 55, 2501704 (2019).

[38] Y. You, X. Chen, X. Zhou, Y. Gu, R. Zhang, F. Pan, and C. Song, Anomalous Hall effect-like behavior with in-plane magnetic field in noncollinear antiferromagnetic $\mathrm{Mn}_{3} \mathrm{Sn}$ films, Adv. Electron. Mater. 5, 1800818 (2019).

[39] A. Markou, J. M. Taylor, A. Kalache, P. Werner, S. S. P. Parkin, and C. Felser, Noncollinear antiferromagnetic $\mathrm{Mn}_{3} \mathrm{Sn}$ films, Phys. Rev. Mater. 2, 051001(R) (2018).

[40] See Supplemental Material at http://link.aps.org/supplemental/ 10.1103/PhysRevB.101.094404 for additional details of the thin-film growth procedure, as well as supporting magnetotransport results.

[41] T. Noll and F. Radu, in Proceedings of MEDSI2016, Barcelona, Spain, September 2016, edited by V. R. W. Schaa (JACoW, Geneva, Switzerland, 2017), p. 370.

[42] P. Carra, B. T. Thole, M. Altarelli, and X. Wang, X-ray Circular Dichroism and Local Magnetic Fields, Phys. Rev. Lett. 70, 694 (1993).

[43] J. M. Taylor, E. Lesne, A. Markou, F. K. Dejene, P. K. Sivakumar, S. Pöllath, K. G. Rana, N. Kumar, C. Luo, H. Ryll, F. Radu, F. Kronast, P. Werner, C. H. Back, C. Felser, and S. S. P. Parkin, Magnetic and electrical transport signatures of uncompensated moments in epitaxial thin films of the noncollinear antiferromagnet $\mathrm{Mn}_{3} \mathrm{Ir}$, Appl. Phys. Lett. 115, 062403 (2019). 